ISSN 1991- 8690

website:http://jsci.utq.edu.iq

الترقيم الدولي 8690 - 1991

Email:utjsci@utq.edu.iq

\title{
Distributed Multipath Routing with Handling of Congestion in TCP/IP Networks using Neural Network
}

\author{
Khulood Ahmed Nassar \\ Lubna Najah Rasoul
}

Computer Science Dept. - University of Basrah - Basrah, Iraq

\begin{abstract}
$\underline{\text { Abstract }}$
Unlike traditional routing schemes that route all traffic along a single path, multipath routing strategies split the traffic among several paths in order to ease congestion. In this paper, one method is proposed to solve multipath routing problem in TCP/IP networks. A feed forward neural network is included at each node of network to make local decision by finding all neighbor nodes for the source node that reach to destination node, also it take in consideration handling of congestion problem. The proposed method is applied for typical examples of computer networks. The recurrent neural network is trained. Results of testing proof on its good performance.
\end{abstract}

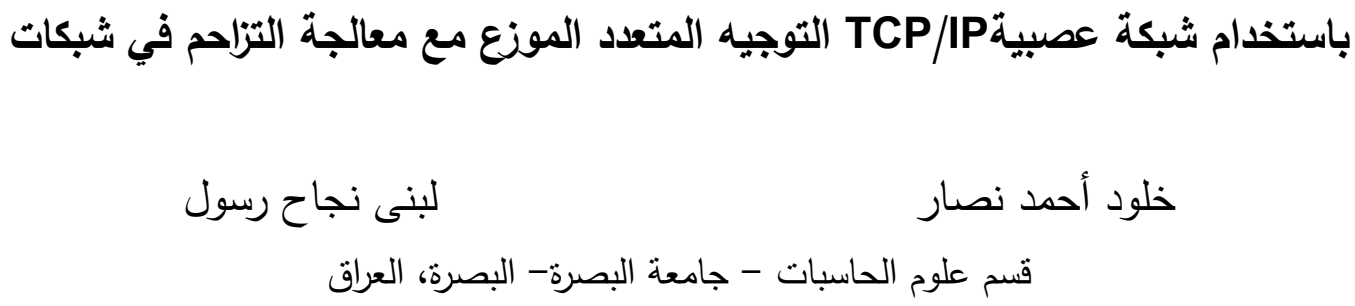

المستخلص

بخلاف مخططات تسيير البيانات التقليدية والتي توجه جميع البيانات في مسلك منفرد، إستراتيجيات إيجاد المسالك المتعددة تفصل الييانات وتوزعها على مساللك متعددة لحل مشكلة التزاحم. في هذا البحث، طريقة واحدة قد اقترحت لحل مشكلة التوجيه المتعدد في شبكات الحاسبات. شبكة عصبية ذات تغذية أمامية في كل نقطة في الثبكة لصنع قرار محلي بواسطة إيجاد جميع مجاورات نقطة المصدر المؤدية إلى نقطة الهدف، وأيضا تأخذ بنظر الاعتبار حل مشكلة التزاحم. الطريقة المقترحة طبقت لأمنلة نموذجية لشبكات الحاسبات. تم تدريب الثبكة العصبية. نتائج الفحص برهنت على حسن أدائها.

\section{Introduction}

Multipath routing constructs multiple paths for a source and destination and provide faulttolerance and reliability [1]. Currently routing algorithms only consider shortest paths between networks, moreover there is a lack of cooperation between congestion control and routing. It has been shown that congestion control has a significant impact on routing, since packet loss will lower the overall performance of the network. Leveraging multiple paths has been a complex problem in communication system, mainly due to out of order packet arrivals, but it has the potential to increase the overall networks throughput significantly at a relatively low cost [2]. The main contribution of this paper is two-fold (1) a simple algorithm to determine multipath routes; (2) a mechanism to forward traffic over multipath routes 
based on delay time of the links and rate of packet dropping of the nodes. The proposed algorithm for finding multiple paths between source and destination node involves examining all neighbor nodes to the destination from the source node, and classifying these neighbors nodes locally depending on its precedence.

\section{Related works}

There are many works to solve the problem of multipath routing in computer networks using neural networks, and algorithms and analysis network protocols. Some of them are:

- G. Xin, Z. Jun, and Z.Tao [3], demonstrated the significant advantage of distributed multipath routing solutions for congestion minimization. Under different network topologies, they approve the better performance of proposed algorithm Distributed -Congestion- Minimized Multipath (D-CMM) by decreasing the congestion factor and increasing the throughput compared to other multipath routing algorithms, and it is more suitable for long distance communication and link dense networks.

- A. Al-shabibi, and B. Martin [2], proposed a novel congestion aware routing protocol. This protocol uses an innovative representation of congestion for routerrouter links. These congestion statistics are the distributed via an aggregation protocol to other routers in the network.

- P.Kinnari [4], proposed different IP and MPLS traffic engineering methods. One of them is (ECMP) that enable the usage of multiple equal cost paths from the source node to the destination node in the network. The purpose of this thesis is to discuss the theoretical and practical issues of ECMP.

- P. Terdal, V. D. Mytri, Damodaram, and Uday. S. B [5], proposed a routing mechanism that can take advantage of the service differentiation offered by Enhanced Distributed Channel Access (EDCA) MAC and the same time overcome its limitation under heavy data. This work measures the available bandwidth of the high priority access categories, energy level and contention level experienced at the intermediate nodes to determine robust paths and divert the audio-video stream along such less congested paths, to ensure better end-to-end delay and throughput.

- W. Yang, X. Yang, G. Liu, and C. Dong [6], presented a Bandwidth Aware Multipath Routing (BAM) protocol, which can find two disjoint parallel paths between source and destination based on the available bandwidth restriction. In BMR, the available bandwidth of nodes is obtained based on a cross-layer mechanism, which can provide a metric for routing discovery.

- K. Valarmath, and Dr. N. Malmurugan [1], proposed a Reliable Multipath Routing (RMR) protocol for 802.16 wireless mesh networks. It consists of a combined routing metric which is based on interference and load on the links. Multiple shortest paths are discovered based on the combined routing metric, the source node chooses the path with the minimum weight value as the primary path. The paths with next minimum weight values are selected as backup paths. Initially the data transmission takes place using the primary path and during any fault, it can be switched over the backup paths.

\section{Multipath routing and congestion in} TCP/IP networks

The handling of congestion in TCP/IP networks based on forward traffic on multiple routes is explained as following:- 


\section{TCP/IP}

Transmission Control Protocol/Internet Protocol (TCP/IP) is like a language that computers speak. It is a set of rules that defines how two computers address each other and send data to each other, also it is a strong, fast, scalable, and efficient suite of protocols [7].

\section{Multipath routing}

Multipath routing constructs multiple paths for a source and destination and provides fault-tolerance and reliability. It has been a complex problem in a communication system, mainly due to out of order packet arrivals, but it has the potential to increase the overall network throughput significantly at a relatively low cost. In multipath routing routers maintain multipath set of two or more paths to each destination subnet $[2,8,9]$.

\section{Congestion}

Congestion is a problem that occurred in shared networks, when multiple users access to the same resources (bandwidth, buffers, and queues). Congestion in a network may occur when the load on the network i.e. the number of packets sent to the network is greater than the capacity of network. Today, most of the congestion control is handled in the transport layer of network communications. The most often used protocol for network transport in today's applications is TCP $[1,10]$.

Ideally, multipath routing claims that each node has more than one route to reach the destination. If one route is failed, another route will be used to keep data communication un inter raptly. So, multipath routing can improve network resilience. On the other side, multiple routes can be used to transmit data concurrently. So, multipath routing can increase bandwidth [11].

In this paper the congestion control will be handled in the network layer (the third layer of TCP/IP layered model) by putting the proposed neural network at each router in the network to determine all neighbor nodes that reach to the destination. Multiple paths are discovered based on the proposed neural network and the source node chooses the best neighbor node (i.e. the node with the minimum weight value) of primary path. The neighbor nodes with next minimum weights values are selected of backup paths. Initially the data transmission takes place using the primary path and during any congestion and fault, it can be switched to the backup paths.

\section{Proposed method}

This paper presents proposed approach based on using neural network to solve the multipath routing problem in a context of computer networks. The neural network of this approach responsible on making routing decision.

The computer networks which are considered in this paper modeled as graphs, where a graph $\mathrm{G}(\mathrm{V}, \mathrm{E})$ is two sets of object, vertices ( or nodes), set V, and edges, set E. A graph is represented with dots or circles (vertices) joined by lines (edges),[12]. Two examples, the first is a 8-node computer network (CN1) shown in Figure (1). While the second is a 14-node (NSFNET topology) computer network (CN2) shown in Figure (2). The imposing values of delay time of the links and rate of packet dropping of neighbor nodes for these two computer networks are shown in Tables (1) and (2), respectively

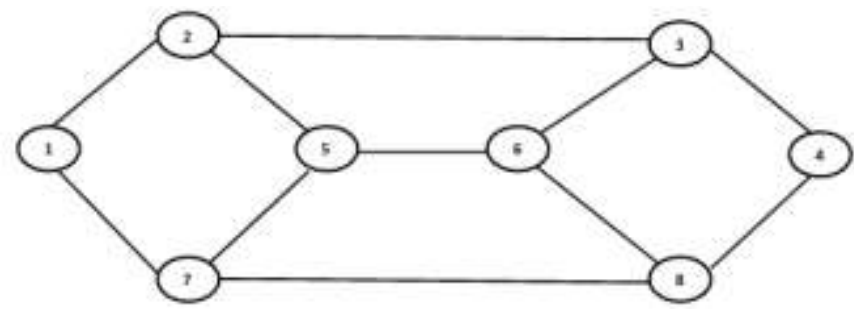

Figure (1) Computer network (CN1) [13] 


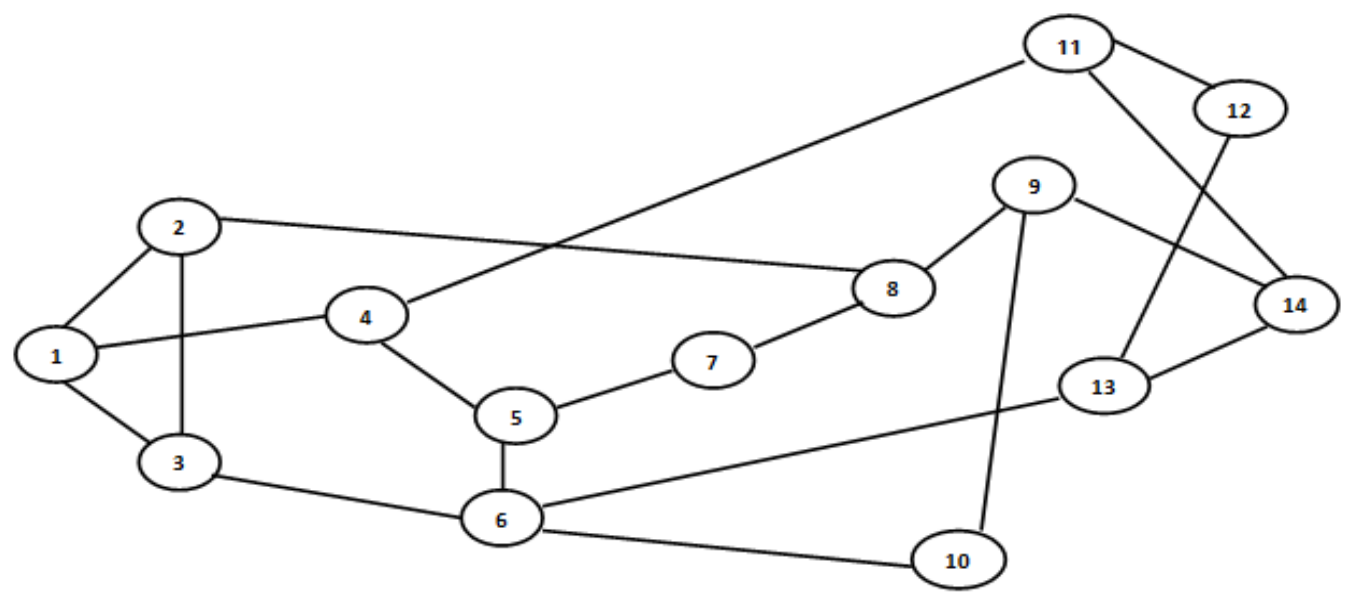

Figure (2) Computer network (CN2) [14]

Table (1) Delay time of link, and rate of packet dropping for computer network (CN1)

\begin{tabular}{|c|c|c|c|}
\hline Link & $\begin{array}{c}\text { Delay time of link } \\
\text { (in second) }\end{array}$ & No. node & $\begin{array}{c}\text { Rate of packet } \\
\text { dropping of neighbor } \\
\text { nodes }\end{array}$ \\
\hline$(1-2)$ & 2 & 1 & 0.07 \\
\hline$(1-7)$ & 6 & 2 & 0.39 \\
\hline$(2-3)$ & 7 & 3 & 0.74 \\
\hline$(2-5)$ & 2 & 4 & 0.88 \\
\hline$(3-4)$ & 3 & 5 & 0.02 \\
\hline$(3-6)$ & 3 & 6 & 0.15 \\
\hline$(5-6)$ & 2 & 7 & 0.30 \\
\hline$(5-7)$ & 1 & 8 & 0.35 \\
\hline$(6-8)$ & 2 & & \\
\hline$(7-8)$ & 4 & & \\
\hline$(8-4)$ & 2 & & \\
\hline
\end{tabular}


Table (2) Delay time of link, and rate of packet dropping for computer network (CN2)

\begin{tabular}{|c|c|c|c|}
\hline Link & $\begin{array}{c}\text { Delay time of link } \\
\text { (in second) }\end{array}$ & No. nodes & $\begin{array}{c}\text { Rate of packet dropping } \\
\text { of neighbor nodes }\end{array}$ \\
\hline$(1-2)$ & 2 & 1 & 0.07 \\
\hline$(1-3)$ & 5 & 2 & 0.39 \\
\hline$(1-4)$ & 7 & 3 & 0.74 \\
\hline$(2-3)$ & 4 & 4 & 0.88 \\
\hline$(2-8)$ & 3 & 5 & 0.02 \\
\hline$(3-6)$ & 1 & 6 & 0.15 \\
\hline$(4-5)$ & 2 & 7 & 0.30 \\
\hline$(4-11)$ & 7 & 8 & 0.35 \\
\hline$(5-6)$ & 2 & 9 & 0.77 \\
\hline$(5-7)$ & 1 & 10 & 0.90 \\
\hline$(6-10)$ & 2 & 11 & 0.55 \\
\hline$(6-13)$ & 6 & 12 & 0.10 \\
\hline$(7-8)$ & 7 & 13 & 0.62 \\
\hline$(8-9)$ & 2 & 14 & \\
\hline$(9-10)$ & 3 & & \\
\hline$(9-14)$ & 3 & & \\
\hline$(11-12)$ & 2 & & \\
\hline$(11-14)$ & 1 & & \\
\hline$(12-13)$ & 2 & & \\
\hline$(13-14)$ & 4 & & \\
\hline
\end{tabular}

\section{Local routing decision}

To solve the multipath routing problem locally, a feed forward neural network (NN) is developed. This neural network has $2 n+1$ inputs, they are destination, $n$ of link delay time and $n$ of rate of packet dropping of neighbor nodes, and $n$ outputs, as shown in figure(3), where $n$ is a number that represent the number of neighbor nodes to the current node (source) that reach to the destination node in the computer network. The output of neural network ordered begins from the best neighbor node, which is the node that has minimum rate of packet dropping, if two neighbor nodes have the same rate of packet dropping, the best neighbor node is the node that connecting with the link has minimum delay time.

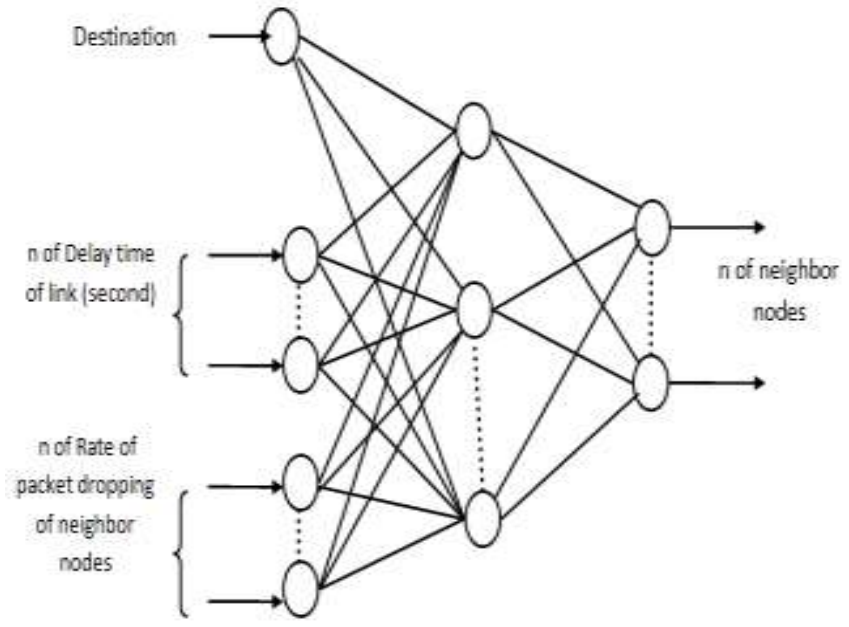

Figure (3) the feed forward neural network (NN)

The computer network includes uch neural network at each node. The snode has information on delay time of links and rate of packet dropping of the 
neighbor nodes to send packet to its neighbor nodes. As a result the neural network determines all neighbor nodes that reach to the destination node, for that, the packet at a node can be arrived to destination node on best path with in minimum rate of packet dropping and minimum delay time on the link. At first, the neural network determine all neighbor nodes, then the node chooses the best neighbor node to sent packet to the destination node, and due to any congestion or fault the node changed to the next best neighbor node, to solve the fault or congestion problem. For example, in the computer network (CN1), if the node 2 has packet to be sent to destination node 4 , then the neural network $(\mathrm{NN})$ at node 2 receives destination 4, delay time of link 2-1, 2-3, and 2-5, and rate of packet dropping for the neighbor nodes $(1,3,5)$. It determines all neighbor nodes to reach node 4 quickly ordered depends on its precedence. The same process is repeated at each node successively till the destination node.

\section{Simulation results}

The neural networks (NN) has been applied to the two examples of the computer networks $(\mathrm{CN} 1$, and $\mathrm{CN} 2$ ) respectively. The implementation has been realized in $\mathrm{C}++$. The activation function used is sigmoid function as in equation:-

$F(x)=1 /\left(1+e^{-\lambda x}\right)$

Where $\lambda$ is size of step, $\lambda \geq 0$ (is taken between 0 and 1). Where real numbers are used to represent their inputs and outputs.

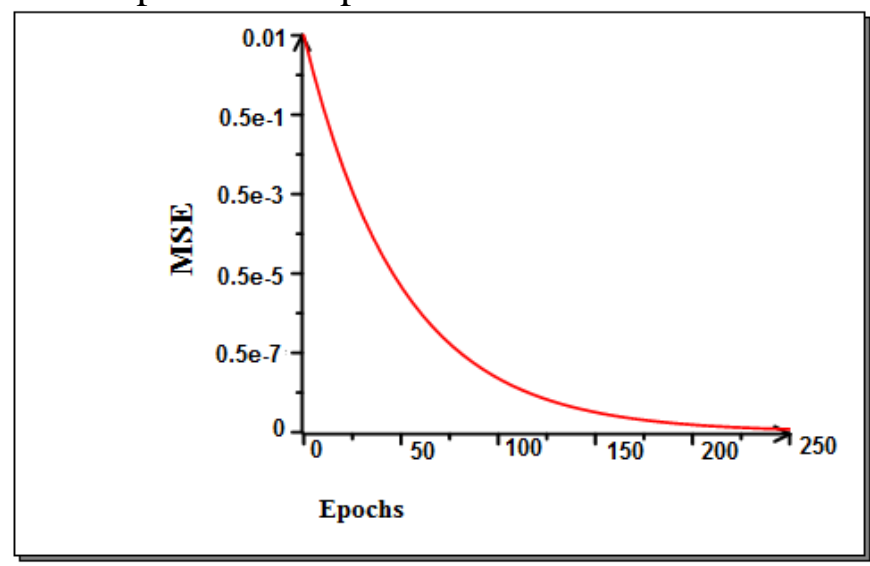

Figure (4) error versus number of epochs of the feed forward neural network (NN) for the computer network (CN1).
The training set and the test set are prepared and the selected neural network is trained to determine all neighbor nodes. In the training stage, random values between $(-0.5-0.5)$ are used as initial weights of connection of the neural network. The neural network (NN) are trained on (30) training sets for each of the computer networks (CN1 and CN2).

In neural network (NN), the training set consists of inputs of the neural network includes (destination, delay time of the links that connect between the current node and its neighbor nodes, rate of packet dropping of neighbor nodes), and all neighbor nodes as output.

In the training stage the value of learning rate is $(0.8)$, and the value of the momentum rate (0.5)for all the computer networks ( $\mathrm{CN} 1$ and $\mathrm{CN} 2)$ are used (are selected by trial and error).

The training is continued until the mean squared error (MSE) become acceptable. When (MSE) decreased, the number of epochs is increased. Figures (4), and (5) view mean squared error versus number of epochs for computer networks $(\mathrm{CN} 1$ and $\mathrm{CN} 2$ ), respectively.

Results of testing are given in Tables (3), and (4), for computer networks (CN1 and CN2). Success rate of testing of the neural network $(\mathrm{NN})$ for the computer networks (CN1 and $\mathrm{CN} 2)$ is shown in Table (5).

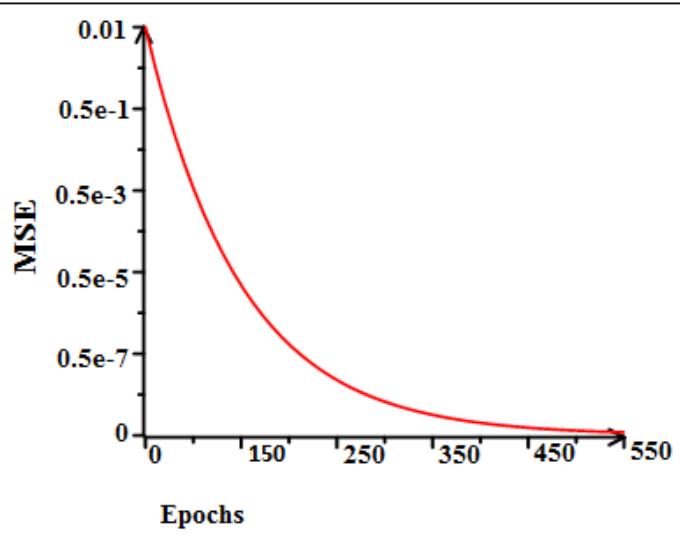

Figure (5) error versus number of epochs of the feed forward neural network (NN) for the computer network (CN2). 
Table (3) some of test result of feed forward neural network (NN) for computer network (CN1)

\begin{tabular}{|l|l|c|c|}
\hline S. & D. & $\begin{array}{c}\text { Real ordering of neighbor node } \\
\text { for the source that access to the } \\
\text { destination }\end{array}$ & The output of the neural network \\
\hline 1 & 4 & {$\left[\begin{array}{ll}7 & 2\end{array}\right]$} & {$\left[\begin{array}{ll}7 & 2\end{array}\right]$} \\
\hline 1 & 8 & {$\left[\begin{array}{ll}7 & 2\end{array}\right]$} & {$\left[\begin{array}{lll}7 & 2\end{array}\right]$} \\
\hline 2 & 6 & {$\left[\begin{array}{lll}1 & 5 & 3\end{array}\right]$} & {$\left[\begin{array}{lll}1 & 5 & 3\end{array}\right]$} \\
\hline 2 & 4 & {$\left[\begin{array}{lll}1 & 5 & 3\end{array}\right]$} & {$\left[\begin{array}{lll}1 & 5 & 3\end{array}\right]$} \\
\hline 3 & 7 & {$\left[\begin{array}{lll}6 & 2 & 4\end{array}\right]$} & {$\left[\begin{array}{lll}6 & 2 & 4\end{array}\right]$} \\
\hline 3 & 8 & {$\left[\begin{array}{lll}6 & 2 & 4\end{array}\right]$} & {$\left[\begin{array}{lll}6 & 2 & 4\end{array}\right]$} \\
\hline 8 & 6 & {$\left[\begin{array}{lll}6 & 7 & 4\end{array}\right]$} & {$\left[\begin{array}{lll}6 & 7 & 4\end{array}\right]$} \\
\hline 5 & 8 & {$\left[\begin{array}{lll}6 & 7 & 2\end{array}\right]$} & {$\left[\begin{array}{lll}6 & 7 & 2\end{array}\right]$} \\
\hline 6 & 4 & {$\left[\begin{array}{lll}5 & 8 & 3\end{array}\right]$} & {$\left[\begin{array}{lll}5 & 8 & 3\end{array}\right]$} \\
\hline 7 & 8 & {$\left[\begin{array}{lll}5 & 1 & 8\end{array}\right]$} & {$\left[\begin{array}{lll}5 & 1 & 8\end{array}\right]$} \\
\hline
\end{tabular}

Table (4) some of test result of feed forward neural network (NN1) for computer network (CN2)

\begin{tabular}{|c|c|c|c|}
\hline S. & D. & $\begin{array}{l}\text { Real ordering of neighbor node for the } \\
\text { source that access to the destination }\end{array}$ & The output of the neural network \\
\hline 1 & 11 & {$\left[\begin{array}{lll}2 & 3 & 4\end{array}\right]$} & {$\left[\begin{array}{lll}2 & 3 & 4\end{array}\right]$} \\
\hline 3 & 4 & {$\left[\begin{array}{lll}1 & 6 & 2\end{array}\right]$} & {$\left[\begin{array}{lll}1 & 6 & 2\end{array}\right]$} \\
\hline 4 & 14 & {$\left[\begin{array}{lll}5 & 1 & 11\end{array}\right]$} & {$\left[\begin{array}{lll}5 & 1 & 11\end{array}\right]$} \\
\hline 5 & 9 & {$\left[\begin{array}{lll}6 & 7 & 4\end{array}\right]$} & {$\left[\begin{array}{lll}6 & 7 & 4\end{array}\right]$} \\
\hline 6 & 9 & {$\left[\begin{array}{llll}5 & 13 & 3 & 10\end{array}\right]$} & {$\left[\begin{array}{llll}5 & 13 & 3 & 10\end{array}\right]$} \\
\hline 7 & 1 & {$\left[\begin{array}{ll}5 & 8\end{array}\right]$} & {$\left[\begin{array}{ll}5 & 8\end{array}\right]$} \\
\hline 8 & 3 & {$\left[\begin{array}{ll}7 & 9\end{array}\right]$} & {$\left[\begin{array}{ll}7 & 9\end{array}\right]$} \\
\hline 9 & 4 & {$\left[\begin{array}{lll}8 & 14 & 10\end{array}\right]$} & {$\left[\begin{array}{lll}8 & 14 & 10\end{array}\right]$} \\
\hline 10 & 1 & {$\left[\begin{array}{ll}6 & 9\end{array}\right]$} & {$\left[\begin{array}{ll}6 & 9\end{array}\right]$} \\
\hline 11 & 1 & {$\left[\begin{array}{lll}12 & 14 & 4\end{array}\right]$} & {$\left[\begin{array}{lll}12 & 14 & 4\end{array}\right]$} \\
\hline 12 & 1 & {$\left[\begin{array}{ll}13 & 11\end{array}\right]$} & {$\left[\begin{array}{ll}13 & 11\end{array}\right]$} \\
\hline
\end{tabular}

Table (5) the success rate of testing the proposed feed forward neural network (NN)

\begin{tabular}{|c|c|c|}
\hline Computer networks & $\begin{array}{c}\text { Success rate of test on trained } \\
\text { sets }\end{array}$ & $\begin{array}{c}\text { Success rate of test on } \\
\text { other sets }\end{array}$ \\
\hline CN1 & $100 \%$ & $94 \%$ \\
\hline CN2 & $100 \%$ & $94 \%$ \\
\hline
\end{tabular}

\section{Conclusions}

This paper introduces the description of proposed method of using neural network for solving the multipath routing problem in computer networks. Local routing decision can be made, when the feed forward neural network is used. The avoiding of congestion node by node is made efficiently. The number of inputs of the proposed feed forward neural networks which determine the 
best neighbor node has no related with size of the computer network. The results obtained during training and testing stages of the proposed neural network show its good performance, in making local multipath routing decision, when it is applied for two different examples of computer network structures.

\section{References}

[1]. K. Valarmath, and Dr. N. Malmurugan, "Reliable Multipath Routing for 802.16 Wireless Mesh Networks", University Chennai, India, Indian Journal of Computer Science and Engineering (IJCSE), Vol.2, No.6, 2012.

[2]. A. Al-shabibi, and B. Martin, "Multi Route A Congestion-Aware Multipath Routing Protocol", University of Heidelberg, International Conference on High Performance Switching and Routing, 2010.

[3]. G. Xin, Z. Jun, and Z.Tao, "A Distributed Multipath Routing Algorithm to Minimize Congestion", University of Aeronautics and Astronautics, 2009.

[4]. M. Sc(Tech.)P.Kinnari, "Equal Cost Multipath Routing in IP Networks", Master thesis, Aalto University, School of Science and technology, 2011.

[5]. S. P. Terdal, Dr. V. D. Mytri, Dr. Damodaram, and Uday. S. B, "Enhanced Multipath Routing with Congestion Avoidance for 802.11E based Mobile Ad Hoc Networks", International Journal of Ad Hoc, Sensor \& Ubiquitous Computing(IJASUC), 2011.

[6]. W. Yang, X. Yang, G. Liu, and C. Dong, "A Bandwidth Aware Multipath Routing Protocol in Mobile Ad Hoc Networks", Jiaotong University, Journal of Computational Information System, 2011.
[7]. A. G. Blank, "TCP/IP Foundations", Sna Francisco, London, 2004, Neil Edde.

[8]. B.L.Mark, and S.Zhang, "A Multipath Flow Routing Approach for Increasing Throughput in the Internet", George Mason University, 2008.

[9]. Prof. S. A. Jain, Mr. A. Bande, Mr. G. deshmukh, Mr. Y. rade, and Mr. M. Sandhanshiv, "An Improvement in Congestion Control using Multipath Routing in MANET", University of Pune, India, International Journal of Engineering Research and Applications(IJERA), Vol.2, No.6, 2012.

[10]. J. A. Bivens, B. K .Szymanski, and M. J. Embrechts, "Network Congestion Arbitration and Source Problem Prediction using Neural Networks", Smart Engineering System Design, 2002.

[11]. B.Dai, H.Lu, Z.Sun, Z.Song, Y.Ma, and J.Su, "MORT: A Technique to Improve Routing Efficiency in fault tolerance Multipath Routing 2009 Fifth International Conference on Mobile Ad-Hoc and Sensor Networks.", National University of Defence Technology,

[12]. N. B. Jarah, "Efficient Routing in Ad-Hoc Wireless Networks using Connected Dominating Set", University of Basra, Journal of Basra Researches ((Sciences)), Vol.38, Num.4.A, 2012.

[13]. H. S. Alshahen "Neural Network Based Routing In Computer Networks" University of Basra, 2009.

[14]. S. Sohn, B. L. Mark, and J. T. Brassil "Congestion Triggered Multipath Routing Based On Shortest Path Information", George Mason University, 2007. 